\title{
Electric-field-induced emergent electrical connectivity in graphene oxide
}

\author{
M. Neek-Amal, ${ }^{1,}{ }^{*}$ R. Rashidi, ${ }^{1}$ Rahul R. Nair, ${ }^{2,3}$ D. Neilson, ${ }^{4}$ and F. M. Peeters ${ }^{2,4}$ \\ ${ }^{1}$ Department of Physics, Shahid Rajaee Teacher Training University 16875-163, Tehran, Iran \\ ${ }^{2}$ National Graphene Institute, University of Manchester, Manchester, M13 9PL, United Kingdom \\ ${ }^{3}$ School of Chemical Engineering and Analytical Science, University of Manchester, Manchester, United Kingdom \\ ${ }^{4}$ Department of Physics, Universiteit Antwerpen, Groenenborgerlaan 171, B-2020 Antwerpen, Belgium
}

(Received 10 December 2018; revised manuscript received 12 February 2019; published 19 March 2019)

\begin{abstract}
Understanding the appearance of local electrical connectivity in liquid filled layered graphene oxide subjected to an external electric field is important to design electrically controlled smart permeable devices and also to gain insight into the physics behind electrical effects on confined water permeation. Motivated by recent experiments [K. G. Zhou et al. Nature (London) 559, 236 (2018)], we introduce a new model with random percolating paths for electrical connectivity in micron thick water filled layered graphene oxide, which mimics parallel resistors connected across the top and bottom electrodes. We find that a strong nonuniform radial electric field of the order $\sim 10-50 \mathrm{mV} / \mathrm{nm}$ can be induced between layers depending on the current flow through the formed conducting paths. The maxima of the induced fields are not necessarily close to the electrodes and may be localized in the middle region of the layered material. The emergence of electrical connectivity and the associated electrical effects have a strong influence on the surrounding fluid in terms of ionization and wetting which subsequently determines the permeation properties.
\end{abstract}

DOI: 10.1103/PhysRevB.99.115425

\section{INTRODUCTION}

Layered dielectrics such as graphene oxide (GO) and other dielectrics are widely used as substrates and gate dielectrics to build different two-dimensional nanoscale devices, e.g., graphene-based vertical heterostructures [1], waveguide connectors [2], and thin film transistors [3]. Specifically, GO, a hydrophilic atomic sheet of graphite with oxygen functionalities, is a graphene-based dielectric which forms a layered assembly with an adjustable interlayer spacing at different humidity [4-6] due to absorbing water. Membrane structures made of GO show an exceptional water permeation and molecular sieving properties [5-7]. Recently, it was found that when a DC bias voltage is applied across the moistened layered porous GO, both molecular permeation and electrical conductivity of the material are affected [8]. Notably, the electric field breakdown $\left(E_{B D}\right)$ strength of dry GO and typical dry layered dielectrics was found to be comparable to that of $\mathrm{SiO}_{2}$ which is typically $E_{B D}=0.1-0.3 \mathrm{mV} / \mathrm{nm}$ [9]. Surprisingly, electric fields of about $10 \mathrm{mV} / \mathrm{nm}$ are able to cause electrical breakdown and subsequently allowed for a precise control of water permeation: from ultrafast permeation to complete blocking. In fact, water fills up the voids of porous layered GO and significantly influences the dielectric properties of the system. In a different experiment it was shown that depending on the low-voltage sign, ion diffusion rates can be reversibly modulated and is anomalously enhanced by a factor of 4-7 within a voltage range of $0.5 \mathrm{~V}$ [11].

In this paper, we introduce a model to investigate the electrical connectivity in moistened GO films. In particular,

*Corresponding author: mehdi.neekamal@gmail.com we focus on the induced electric field around the generated conducting routes.

\section{MODEL}

When an external voltage is applied, it was experimentally found that several conducting paths are formed between successive layers of the GO membrane $[8,10]$ that link the top and the bottom electrodes, see Fig. 1. Our model is constructed as a combination of series and parallel arrangements of resistors. These can be either metallic nanowires such as $(n, 0)$ zig-zag nanotubes, polycyclic aromatic hydrocarbons, or amorphous carbon paths in GO, which are connected to each other and subsequently connect the top and bottom electrodes obeying Ohm's law. Our example here is the insulating-to-conducting transition in GO laminates upon the application of an external electric field.

Recently, we measured the electrical conductivity of GO membranes subjected to external bias voltage that induces an electric field perpendicular to the layers [8]. The estimated current through each individual filament, for electrically conductive GO layers, was found to be about $1 \mathrm{nA}$ for a $1 \mathrm{~V}$ applied potential [8].

To gain insight into the unexplored phenomenon of the emergence of global connectivity perpendicular to the basal planes of layered GO, it is fruitful to overlook details and to ask for the simplest model which reproduces the essential physical features.

Therefore, we will neglect the microscopic details of the membrane and conducting filaments. The observed selfhealing phenomenon in Ref. [8] indicates clearly that the breakdown mechanism of moistened layered GO is controlled by the carbon chains in the system. The carbon chains may be more important in determining the breakdown strength than the layered GO (and water) itself [12]. For instance, a 


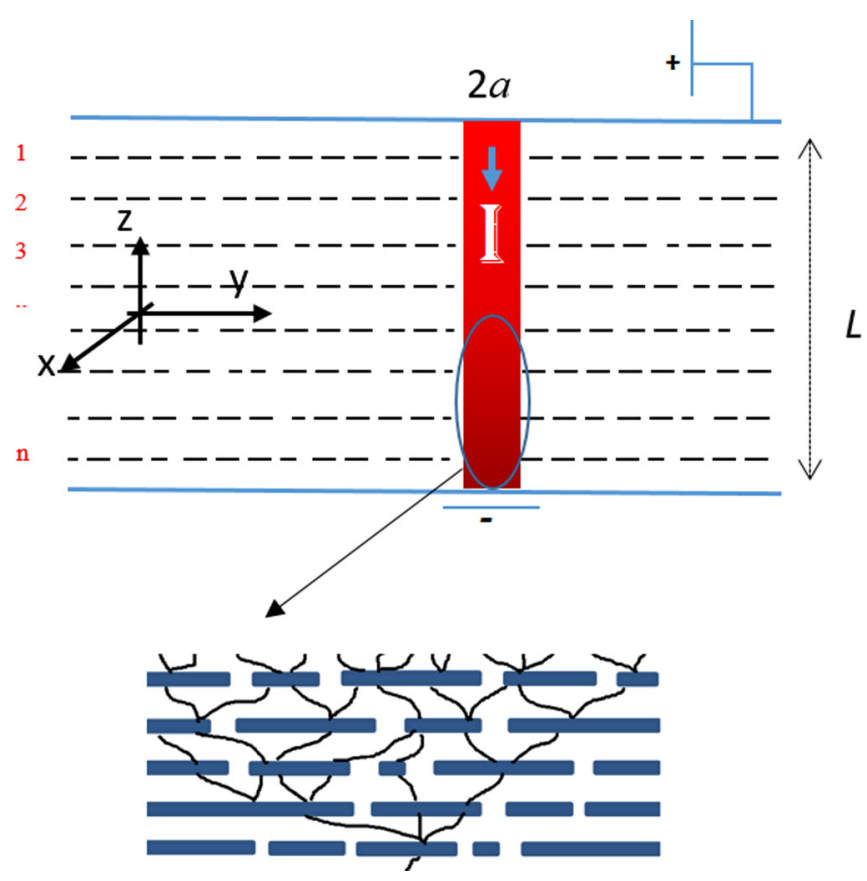

FIG. 1. A schematic of the emergence of global electrical connectivity in a porous layered GO device with thickness $L$ and " $n$ " layers. The middle red wire with radius " $a$ " is the simplest representation of a conducting route. The bottom figure represents the formed conducting filaments between the layers. The parallel resistors model causes the resistivity to be nonuniform across the membrane.

sample exposed to zero humidity did not show any evidence of formation of electrically conducting filaments even up to $50 \mathrm{~V}$ [8]. Moreover, the electric breakdown strength $\left(E_{B D}\right)$ of water is around $30 \mathrm{mV} / \mathrm{nm} \mathrm{[13]} \mathrm{and} \mathrm{that} \mathrm{of} \mathrm{the} \mathrm{dry} \mathrm{layered}$ dielectric is larger than $100 \mathrm{mV} / \mathrm{nm}$, while the measured $E_{B D}$ in recent experiment was found to be about $\sim 2 \mathrm{mV} / \mathrm{nm}$ [8]. These findings lead us to conclude that the presence of interconnects (carbon chains or other contaminants within the layered dielectrics [14]) facilitates electrical conductivity. The interconnect carbon chains might be polarized and experience a force in a nonuniform field between the layers. The force moves the interconnected particles toward the maximum field region within each layer and align them with the field. Eventually they will link the successive layers (such that far from the cathode the number of aligned particles is larger), yielding an electrical current that passes across the membranes. Notice that the in-plane IV characterization in the experiment of Ref. [8] results in highly resistive GO confirming that the conducting filaments are oriented to the out-of-plane direction parallel to the applied external field.

Reality may be more complex because of the complex nature of the microscopic structure of common layered dielectrics such as GO, the unusual porosity, and the effects of metal-liquid interfaces. Nevertheless, we assume that similarly to electrical treeing phenomenon (in the light of random branching), several parallel resistors may be formed between successive layers close to one of the electrodes, and a few formed close to the other electrode (see bottom panel of Fig. 1). This last assumption gives a higher probability of finding a global connectivity. Because of the porosity in the
TABLE I. The number we used for the parameters of the model which correspond to the experimental data of Ref. [8].

\begin{tabular}{lcc}
\hline \hline Parameter & Experiment [8] & Present work \\
\hline Thickness $(\mu \mathrm{m})$ & $1.0,5.0$ & $1.0,5.0$ \\
Voltage $(\mathrm{V})$ & $0.0-4.0$ & $0.0-4.0$ \\
Resistance $(\Omega)$ & $100-200$ & $100-200$ \\
Electric field $(\mathrm{mV} / \mathrm{nm})$ & $1-10.0$ & $1.0-50.0$ \\
Conducting route size $(\mathrm{nm})$ & $5-100.0$ & $5-100.0$ \\
\hline \hline
\end{tabular}

system, finding a straight bridge between the bottom and top electrode is less plausible.

For the setup shown in Fig. 1, the number of parallel resistors increases nonlinearly with increasing $z$ and they are taken for simplicity to be in a cylindrical region of radius $a$ and length $L$, i.e., a conductive wire (see red column in Fig. 1). This simplifies our model. There are a few global bridges (red wire) distributed over the surface of the metallic electrodes [8]. In other words, $L$ is the thickness of the layered GO and $a \sim 5-10 \mathrm{~nm}$ is the size of the portion within which the connectivity occurs (typically $a / L \sim 0.001-0.01$ [15]). We assume $L=n_{0} \times d$ where " $d$ " is the interlayer distance between " $n_{0}$ " number of layers. The wire carries a constant stationary current $I$, see Fig. 1 . Two infinite parallel metallic electrodes are connected by the conducting wire. In Table I we list the model parameters we used that correspond to the experimental data of Ref. [8].

For simplicity, we assume that the wire is an Ohmic resistor with a nonuniform electrical resistivity varying exponentially along its axis $z$, i.e., $\varrho=\varrho_{0} e^{-\alpha z / L}$. This assumption expresses the fact that by increasing the number of conducting filaments close to one electrode, the resistivity should exponentially decay (increase) for $\alpha>0(\alpha<0)$. Then the resistance of the wire as a function of $z$ is given by,

$$
R(z) \simeq \frac{L \varrho_{0}}{\pi \alpha a^{2}}\left(1-e^{-\alpha z / L}\right) .
$$

Here $\alpha$ is the key parameter in our model and controls the degree of multiplication (treeing). Thus $\alpha$ determines the branching pattern of conducting filaments and controls the distribution of conducting filaments. If we reverse the pattern shown in bottom panel in Fig. 1 and fix the $z$-direction as it is for the current representation in Fig. 1, $\alpha$ should be negative. The simple case $\alpha \cong 0$ gives a constant resistivity $\varrho_{0}$ and the familiar resistance $R(z)=\frac{\varrho_{0} z}{\pi a^{2}}$. The larger $\alpha$, the larger the number of parallel resistors close to one of the electrodes and consequently the larger is the degree of branching multiplication. Thus exponential resistivity provides an accurate description of the main features of the branching pattern of the conducting filaments.

Using Eq. (1), we can estimate the total resistance for a given $\alpha$ as $R_{T}=R(L, \alpha)=\frac{L \varrho_{0}}{\pi \alpha a^{2}}\left(1-e^{-\alpha}\right)$. Thus, to find a reasonable range for the $\alpha$ parameter relevant to the experimental data, first we note that the maximum electric current and voltage in the experiment are $20 \mathrm{~mA}$ and $4 \mathrm{~V}$ [8], respectively, resulting in $R(L, \alpha) \preceq 200 \Omega$. Using the relation for $R(L, \alpha)$ we obtained, a typical resistivity in the experimental sample with $L=1 \mu \mathrm{m}$ and $a=10 \mathrm{~nm}$ is given by $\varrho_{0} \preceq 2 \pi \times 10^{-8} \Omega$.m. Second, to estimate $\alpha$, we solve the 
TABLE II. Some possible values for the parameters $\alpha$ as obtained by solving Eq. (2) for various parameters in the model.

\begin{tabular}{ccccc}
$R(\Omega)$ & $L(\mu \mathrm{m})$ & $\varrho_{0}\left(10^{-9} \Omega . m\right)$ & $a(\mathrm{~nm})$ & $\alpha$ \\
\hline 100 & 1 & 1 & 5 & 0.50 \\
100 & 1 & 2 & 5 & 2.28 \\
100 & 1 & 2 & 6 & 1.27 \\
100 & 1 & 2 & 7 & 0.54 \\
200 & 5 & 1 & 5 & 3.02 \\
200 & 5 & 1 & 6 & 1.87 \\
200 & 5 & 1 & 7 & 1.06 \\
200 & 5 & 1 & 8 & 0.45 \\
200 & 5 & 2 & 5 & 3.12 \\
200 & 5 & 2 & 6 & 4.36 \\
200 & 5 & 2 & 7 & 3.10 \\
200 & 5 & 2 & 8 & 2.21 \\
200 & 5 & 2 & 9 & 1.54 \\
200 & 5 & 2 & 10 & 1.01 \\
\hline \hline
\end{tabular}

following equation:

$$
\frac{R_{T} \pi a^{2}}{L}=\frac{1-e^{-\alpha}}{\alpha} .
$$

Solving Eq. (2) for $\alpha$ by using typical values for the resistivity, e.g., $\varrho_{0}=[1-6] \times 10^{-9} \Omega . m$ and for $a=[5-10] \mathrm{nm}$, we are able to determine $\alpha$. Some of the resulting values for $\alpha$ for the various parameters of the model are listed in Table II. We conclude that $\alpha$ values smaller than 5.0 are relevant to the experimental data. The number of filaments $n_{0}$ inside a conducting route with radius $a$ can be estimated as $n_{0}=$ $\frac{\Delta R(z=L)}{\Delta R(z=0)} \cong e^{\alpha}$. For $\alpha=5$ and $a=5 \mathrm{~nm}$ this yields an area density of conducting filaments of $2.0 \mathrm{~nm}^{-2}$.

Furthermore, atomic force microscope (AFM) images confirm the filament formation in conducting GO membranes. A typical Peak Force Tunneling AFM current image of a GO membrane after filament formation exfoliated on a gold-thinfilm-coated silicon substrate is shown in Fig. 1(e) of Ref. [8]. In our model, the high resistance of small pieces (close to the cathode electrode) is due to the lower number of parallel filaments (as compared to the other electrode). This is an essential feature of our proposed electrical treeing model. The electric current was reported to be about $1 \mathrm{~mA}$ for a thickness $L=1 \mu \mathrm{m}[8]$.

\section{RESULTS}

\section{A. Electric field}

In Fig. 2(a) we depict the variation of the resistivity $\varrho$ by the solid line on a semi-log scale. Keeping the bottom and top electrodes at potential 0 and $V_{0}$, respectively (which is taken large enough to generate a global electrical connectivity), the potential on the surface of the resistor and the stationary electric current flowing through the resistor become $V_{0}\left(\frac{e^{-\alpha z / L}-1}{e^{-\alpha}-1}\right)$ and $\frac{\pi \alpha a^{2} V_{0}}{L e_{0}\left(1-e^{-\alpha}\right)}$, respectively. Straightforward calculations using cylindrical coordinates with incorporating proper boundary conditions (see the Appendix) of the Laplace
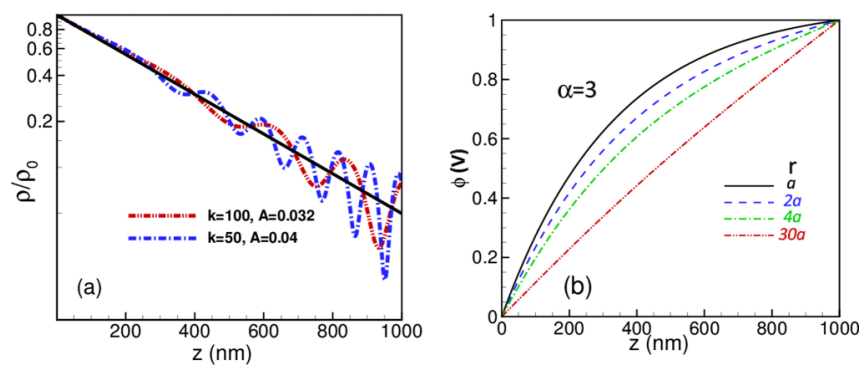

FIG. 2. (a) The variation of $\varrho / \varrho_{0}$ with $z$. The solid line refers to no sinusoidal variation while the two dashed curves are the relative resistivity when a sinusoidal oscillation is added. (b) The variation of electric potential around the conducting cylinder at four different radial distances, i.e., $r=a, 2 a, 4 a, 30 a$ for $\alpha=3.0, a=10 \mathrm{~nm}$, and $L=1000 \mathrm{~nm}$.

equation yield the electric potential outside the wire as

$$
\Phi(r, z)=\frac{V_{0}}{L} z+\sum_{n=0}^{\infty} B_{n} \sin (n \pi z / L) \kappa(r, n),
$$

where $\kappa(r, n)=\frac{K_{0}(n \pi r / L)}{K_{0}(n \pi a / L)}$ and $B_{n}=\frac{2 \alpha^{2}\left((-1)^{n}-e^{\alpha}\right) V_{0}}{n \pi\left(1-e^{\alpha}\right)\left(n^{2} \pi^{2}+\alpha^{2}\right)}$. Here $K_{0}$ denotes the modified Bessel function of the second kind. From Eq. (3) one might deduce that the net electric field is equal to $V_{0} / L$ independent of $\alpha$ and that the contribution of the second term is negligible. However, we will show that, depending on the value of $\alpha, a$, and the distance $r$, the electric field resulting from the second term can be in fact much larger than $V_{0} / L$.

In Fig. 2(b) we depict the variation of $\Phi(r, z)$ versus $z$ for four different radial distances, where we took $\alpha=3$. It is seen that far from the cylinder $(r=30 a)$ we obtain the expected linear behavior of $\Phi(r, z) \propto z$, i.e., the electric potential drops linearly between two parallel plates. In other words the second term in Eq. (3) is negligible. However, on the surface of the cylinder (where $r=a$ ), the electric potential shows a parabolic behavior, leading to an unexpected variation of the radial electric field around the column (see below).

Surprisingly, the total electric field along the wire has a local maximum when $|\alpha|>0$. The maximum occurs at about $Z_{\max } \approx L / 2$ for $\alpha \sim|1|$ and is larger (smaller) for larger (smaller) $|\alpha|$ (see Fig. 3). Notice that near the wire, $r \sim 2 a$, the ratio $E_{r} / E_{z}$ is about 10 , implying that the major contribution is due to the radial component. For larger $\alpha$, a stronger electric field is induced around the wire, e.g., for $\alpha=5$ the total electric field is found to be about $50 \mathrm{mV} / \mathrm{nm}$ where $V_{0}=4 \mathrm{~V}$. In Fig. 3(a), we give a two-dimensional (2D) contour plots of the variation of $E_{t}$ versus $\alpha$ and $z$ and in Fig. 3(b), we show the variation of $E_{t}$ with $a$ and $z$. For smaller $a$ we obtain larger fields. This is due to charge accumulation on the surface of the cylindrical wire. For larger $|\alpha|(\sim 5)$, the maximum is closer to one of the electrodes (depending on the polarity of the applied voltage), but not on the electrodes, and consequently the charge is accumulated close to one of the electrodes.

Figure 4(a) shows the variation of the position of the total electric field along the wire $Z_{\max }$ with $\alpha$ for $V_{0}=1 \mathrm{~V}$ and $a=10 \mathrm{~nm}$.

The appearance of peaks around the middle of the column and the key role of parameter $\alpha$, which determines the percolating path, indicates that the mechanism behind electrical 

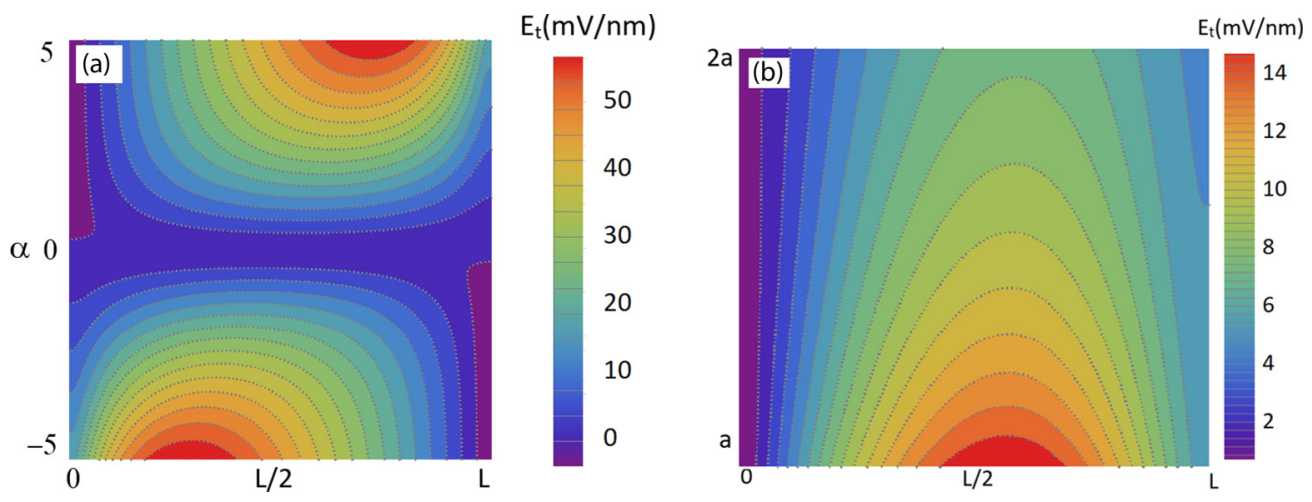

FIG. 3. (a) Contour plot of total electric field on the surface of a conducting wire $(r=a=10 \mathrm{~nm})$ along the wire as function of $\alpha$ for $V_{0}=4 \mathrm{~V}$. (b) 2D-plot of total electric field along wire as function of radius of wire $a$ for $\alpha=1$ and $V_{0}=4 \mathrm{~V}$.

conductivity, of, for example, GO membranes, is different from that of other dielectrics such as the mechanism of lattice distortion by defects in $\mathrm{SiO}_{2}$ and those models in which the anode electrode plays an important role in the electric breakdown $[16,17]$. It is interesting to note that, in addition to the radial symmetry, for a given $r$ we have $E_{t}(z, \alpha)=$ $E_{t}(L-z,-\alpha)$. This is a consequence of the asymmetry in polarity of the external voltage. A similar symmetry exists in the 2D plots shown in Fig. 3.

\section{B. Electric charge}

The surface charge density can be found using $\sigma_{x}=$ $(4 \pi)^{-1} E_{x}(r, z)$, where on the resistor $E_{x}=E_{r=a}$ and on the bottom and top plates $E_{x}=E_{z=0}$ and $E_{z=L}$, respectively. A positive $\alpha$ induces positive charges on the surface of the cylinder. This is due to the nonconstant resistivity of the wire. Notice that a volume charge density is also induced inside the wire which originates from a variation of the resistance inside the wire. The volume charge density can be calculated using $\rho=-\frac{\nabla^{2} \Phi(r, z)}{4 \pi}$. However, we found that the total charge inside the wire $\left(Q_{v}=\pi a^{2} \int_{0}^{z} \rho d z\right)$ is negligible as compared to the surface charge $\left(Q_{v} \simeq 0.05 Q_{s}\right)$. For this reason, we show in

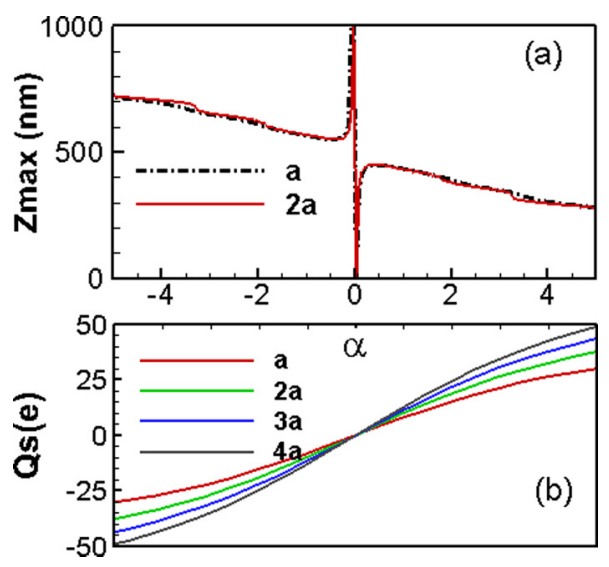

FIG. 4. The variation of (a) $Z_{\max }$ and (b) $Q_{s}$ with $\alpha$ for different wire radius.
Fig. 4(b) only the induced surface charge

$$
Q_{s}=\frac{-8 \epsilon a V \alpha^{2}}{\pi^{2} \tan h(\alpha / 2)} \sum_{n=0}^{\infty} \frac{\kappa_{1}(a, 2 n+1)}{(2 n+1)\left(\frac{\alpha^{2}}{\pi^{2}}+(2 n+1)^{2}\right)},
$$

for four different $a$ values, with $\kappa_{1}(r, n)=\frac{K_{1}(n \pi r / L)}{K_{0}(n \pi a / L)}$. For a wire with effective radius of about $10 \mathrm{~nm}$, the total charge on the surface for $\alpha \sim 3$ is found to be about $20 \mathrm{e}$.

Finally, to include in a simple way, the intrinsic random nature of the emergence of electrical connectivity in our model, we added a sine function to the resistivity, i.e., $\varrho / \varrho_{0}=A \sin (\pi z / k L)+e^{-\alpha z / L}$. In Fig. 2(a) we depict three resistivities in semi-log scale with respect to $z$ with $A=0$, $0.032,0.04$ and $k=0,100,50$, which are represented by a solid line and two dashed lines, respectively. We see that the effects of adding these sinusoidal functions are small. Hence, the main message and the quantitative results are general.

\section{DISCUSSION}

We presented a theory for the electrical connectivity through GO which models the experimentally observed conducting filaments formed between successive layers of GO membranes. Since the electric breakdown strength of water is equal or larger than $10 \mathrm{mV} / \mathrm{nm}[8,13]$, the resulting strong radial electric field around the conducting filaments (up to $50 \mathrm{mV} / \mathrm{nm}$ ), will influence the surrounding water in terms of ionizing the water, and subsequently it will change its permeability. By increasing the electric field, the concentration of $\mathrm{H}_{3} \mathrm{O}^{+}$and $\mathrm{OH}^{-}$ions increases. The larger $V_{0}$ and $\alpha$, the larger the electric field. This is consistent with recent experiments [8]: a strong electric field can dissociate water in the interlayer channels of the porous membranes into $\mathrm{OH}^{-}$and $\mathrm{H}_{3} \mathrm{O}^{+}$ions according to the hydration effect

$$
(\mu+v+2) \mathrm{H}_{2} \mathrm{O} \rightleftarrows \mathrm{OH}^{-} \cdot v\left(\mathrm{H}_{2} \mathrm{O}\right)+\mathrm{H}_{3} \mathrm{O}^{+} \cdot \mu\left(\mathrm{H}_{2} \mathrm{O}\right),
$$

where $\mu$ and $\nu$ determine the degree of hydration. In fact, the dissociated water molecules (yielding the localization of $\mathrm{H}_{3} \mathrm{O}^{+}$and $\mathrm{OH}^{-}$ions inside the channels) quickly disperse within the layers and react with GO. Also the formation of large hydrated clusters due to strong interactions between $\mathrm{H}_{3} \mathrm{O}^{+}$and $\mathrm{OH}^{-}$ions and surrounding water molecules, cause local blockage. This will happen for many channels, in particular at the entrance of the channels. 
It is also important to note that in GO, a strong electric field can change the local arrangement of the functional groups attached on the surface of GO. The electrical breakdown of GO membranes fully recovers its insulating properties once the current through the conducing channel has been externally interrupted (self-healing effect) [8]. This also causes an essential difference between the electric breakdown of layered materials and those found in usual dielectric materials which are characterized by an irreversible response to an external electric field.

\section{SUMMARY}

In summary, we have shown that a strong radial electric field is induced around the electrical conductor between the layers that influences the intercalated liquid in the layers. The strength of the field depends on many parameters including the applied voltage, branching pattern of the emerging conducting filaments, and the distance between the layers. The induced charges and corresponding strong radial electric fields are found to be about $10-100 \mathrm{e}$ and $10-50 \mathrm{mV} / \mathrm{nm}$, respectively, and depend significantly on the percolating path parameter (i.e., the $\alpha$ ). Depending on the applied external voltage and the created branching pattern in the distribution of conducting filaments, the induced electric field around the conducting wire can reach as high as $50 \mathrm{mV} / \mathrm{nm}$. This field is localized around the wire and can alter the surrounding liquid.

Our study opens a new avenue to investigate connectivity of wet-layered dielectric materials that have planar controllable resistivity. It is of importance to predict, control, and minimize the occurrence of electric breakdown in a wide variety of porous layered dielectrics. In particular, since GO membranes with a narrow channel-size distribution are useful materials for ionic and molecular sieving, it is important to understand the effects of local induced electric fields on the liquid between the layers. Our study sheds new light on electrical connectivity of layered dielectrics, showing that it can be linked to percolating properties and the microscopic details of conducting filaments that interconnect the layers.

\section{ACKNOWLEDGMENTS}

This work was supported by the Flemish Science Foundation (FWO-Vl) and the Methusalem program.

\section{APPENDIX}

Consider two infinite parallel metallic electrodes connected by a cylindrical current-carrying resistor of radius $a$ (Fig. 1). The appropriate azimuthally symmetric DirichletGreen function that solve Laplace equation in the space outside the resistor and between the two plates is $[18,19]$

$$
\begin{aligned}
G_{D}\left(r, z ; r^{\prime}, z^{\prime}\right)= & \frac{4}{L} \sum_{n=1}^{\infty} \sin (n \pi z / L) \sin \left(n \pi z^{\prime} / L\right) K_{0}\left(n \pi r_{>} / L\right) \\
& \times\left\{I_{0}\left(n \pi r_{<} / L\right)-\frac{I_{0}(n \pi a / L) K_{0}\left(n \pi r_{<} / L\right)}{K_{0}(n \pi a / L)}\right\},
\end{aligned}
$$

where $L$ is the distance between the two plates and $I_{0}$ and $K_{0}$ denote the modified Bessel function of the first and second kind, respectively, where $r_{>}=\max \left(r, r^{\prime}\right)$ and $r_{<}=\min \left(r, r^{\prime}\right)$. In the absence of any electric charge, the potential for $r>a$ and $0<z<L$ is given by

$$
\Phi(r, z)=-\frac{1}{4 \pi} \oint \Phi\left(r^{\prime}, z^{\prime}\right) \frac{\partial G_{D}}{\partial n^{\prime}} d S^{\prime},
$$

where the integration is taken over the boundary surfaces and $\frac{\partial}{\partial n^{\prime}}$ represents the normal derivative at the boundary surfaces. Taking the Wronskian of $I_{0}$ and $K_{0}$ into account, the radial derivative of $G_{D}$ at $r=a$ can be expressed as

$$
\begin{aligned}
\left(\frac{\partial G_{D}}{\partial n^{\prime}}\right)_{r^{\prime}=a} & =-\left(\frac{\partial G_{D}}{\partial r^{\prime}}\right)_{r^{\prime}=a} \\
& =-\frac{4}{a L} \sum_{n=1}^{\infty} \sin (n \pi z / L) \sin \left(n \pi z^{\prime} / L\right) \kappa(r, a) .
\end{aligned}
$$

Suppose that the cylindrical resistor is an Ohmic resistor with a nonuniform electrical resistivity varying exponentially along the $z$ axis, namely $\varrho=\varrho_{0} e^{-\alpha z / L}$, then the resistance of the resistor as a function of $z$ is given by

$$
R(z)=\int_{0}^{z} \frac{\varrho\left(z^{\prime}\right) d z^{\prime}}{\pi a^{2}}=\frac{L \varrho_{0}}{\pi \alpha a^{2}}\left(1-e^{\alpha z / L}\right),
$$

where $\alpha$ is a dimensionless constant. Keeping the bottom and top electrodes at potential 0 and $V_{0}$, respectively, the potential on the surface of the resistor and the electric current flowing through the resistor in the stationary state, respectively, become

$$
\Phi(r=a, z)=V_{0}\left(\frac{1-e^{-\alpha z / L}}{1-e^{-\alpha}}\right)
$$

and

$$
I=\frac{V_{0}}{R(z=L)}=\frac{\pi \alpha a^{2} V_{0}}{L \varrho_{0}\left(1-e^{-\alpha}\right)} .
$$

A straightforward calculation using Eqs. (A2), and (A3) and the boundary condition (A5) yields the solution

$$
\Phi(r, z)=\frac{V_{0}}{L} z+\sum_{n=0}^{\infty} B_{n} \frac{K_{0}(n \pi r / L)}{K_{0}(n \pi a / L)} \sin (n \pi z / L),
$$

where

$$
\begin{aligned}
B_{n} & =\frac{2 V_{0}}{L} \int_{0}^{L} \sin \left(n \pi z^{\prime} / L\right)\left(\frac{1-e^{-\alpha z^{\prime} / L}}{1-e^{-\alpha}}-\frac{z^{\prime}}{L}\right) d z^{\prime} \\
& =\frac{2 \alpha^{2}\left((-1)^{n}-e^{\alpha}\right) V_{0}}{n \pi\left(1-e^{\alpha}\right)\left(n^{2} \pi^{2}+\alpha^{2}\right)} .
\end{aligned}
$$

To avoid the necessity of integration over the plates, we add and subtract from the potential the linear term $\frac{V_{0} z}{L}$, which satisfies the Laplace equation and the boundary condition at $z=0$ and $z=L$. Then the components of the electric field can be determined as

$$
E_{r}=-\frac{\partial \Phi}{\partial r}=\sum_{n=0}^{\infty} \frac{n \pi B_{n}}{L} \frac{K_{1}(n \pi r / L)}{K_{0}(n \pi a / L)} \sin (n \pi z / L),
$$


and

$$
E_{z}=-\frac{\partial \Phi}{\partial z}=-\frac{V_{0}}{L}-\sum_{n=0}^{\infty} \frac{n \pi B_{n}}{L} \frac{K_{0}(n \pi r / L)}{K_{0}(n \pi a / L)} \cos (n \pi z / L) .
$$

The surface charge densities on the resistor and on the bottom and top plates, respectively, are

$$
\begin{aligned}
\sigma_{r} & =\frac{E_{r}(r=a, z)}{4 \pi} \\
& =\sum_{n=0}^{\infty} \frac{n B_{n}}{4 L} \frac{K_{1}(n \pi a / L)}{K_{0}(n \pi a / L)} \sin (n \pi z / L), \\
\sigma_{b} & =\frac{E_{z}(r>a, z=0)}{4 \pi} \\
& =-\frac{V_{0}}{4 \pi L}-\sum_{n=0}^{\infty} \frac{n B_{n}}{4 L} \frac{K_{0}(n \pi r / L)}{K_{0}(n \pi a / L)}
\end{aligned}
$$

and

$$
\begin{aligned}
\sigma_{t} & =-\frac{E_{z}(r>a, z=L)}{4 \pi} \\
& =\frac{V_{0}}{4 \pi L}+\sum_{n=0}^{\infty} \frac{\left(-1^{n}\right) n B_{n}}{4 L} \frac{K_{0}(n \pi r / L)}{K_{0}(n \pi a / L)} .
\end{aligned}
$$

The total induced charge is given by

$$
Q=\pi a \int_{0}^{z} \sigma_{r} d z+\pi a^{2} \int_{0}^{z} \rho d z
$$

where the first term gives the surface charge and the second term gives the volume charge. After performing the integrals we find

$$
\begin{aligned}
Q_{s} & =2 \pi a \int_{0}^{z} \sigma_{r} d z \\
& =\frac{-8 \epsilon a V \alpha^{2}}{\pi^{2} \tan h(\alpha / 2)} \sum_{n=1 \text { (odd) }}^{\infty} \frac{\kappa_{1}(a, n)}{n\left(\frac{\alpha^{2}}{\pi^{2}}+n^{2}\right)} .
\end{aligned}
$$

[1] C. Dean, A. F. Young, L. Wang, I. Meric, G.-H. Lee, K. Watanabe, T. Taniguchi, K. Shepard, P. Kim, and J. Hone, Solid State Communications 152, 1275 (2012).

[2] K. Zhang, F. Meng, Q. Wu, J.-H. Fu, and L.-W. Li, Europhys. Lett. 99, 47008 (2012).

[3] C.-R. Park and J.-H. Hwang, Ceramics Int. 40, 12917 (2014).

[4] T. Yang, H. Lin, X. Zheng, K. P. Loh, and B. Jia, J. Mater. Chem. A 5, 16537 (2017).

[5] J. Abraham, K. S Vasu, C. D. Williams, K. Gopinadhan, Y. Su, C. T. Cherian, J. Dix, E. Prestat, S. J. Haigh, I. V. Grigorieva, P. Carbone, A. K. Geim, and R. R. Nair, Nat. Nanotechnology 12, 546 (2017).

[6] B. Mi, Sicence 343, 740 (2014).

[7] R. R. Nair, H. A. Wu, P. N. Jayaram, I. V. Grigorieva, and A. K. Geim, Science 335, 442 (2012).

[8] K.-G. Zhou, K. S. Vasu, C. T. Cherian, M. Neek-Amal, J. C. Zhang, H. Ghorbanfekr-Kalashami, K. Huang, O. P. Marshall, V. G. Kravets, J. Abraham, Y. Su, A. N. Grigorenko, A. Pratt, A. K. Geim, F. M. Peeters, K. S. Novoselov, and R. R. Nair, Nature (London) 559, 236 (2018).
[9] B. Standley, A. Mendez, E. Schmidgall, and M. Bockrath, Nano Lett. 12, 1165 (2012).

[10] S. Qin, J. Zhang, D. Fu, D. Xie, Y. Wang, H. Qian, L. Liu, and Z. Yu, Nanoscale 4, 6658 (2012).

[11] C. Cheng, G. Jiang, G. P. Simon, J. Z. Liu, and D. Li, Nat. Nanotechnol. 13, 685 (2018).

[12] I. Balygin, Electric Strength Of Liquid Dielectrics (Foreign Technology Division Wright-Patterson Air Force Base, Ohio, 1972).

[13] M. Kristiansen, High Voltage Water Breakdown Studies, (Texas Tech University Pulsed Power Lab, Lubbock, TX, 1998).

[14] C. Van Dyck, T. J. Marks, and M. A. Ratner, ACS Nano 11, 5970 (2017).

[15] J. J. O’DWyer, J. Adv. Phys. 7, 349 (1958).

[16] G. A. Vorobev, S. G. Ekhanin, and N. S. Nesmelov, Phys. Solid State 47, 1083 (2005).

[17] A. Padovani, D. Z. Gao, A. L. Shluger, and L. Larcher, J. Appl. Phys. 121, 155101 (2017).

[18] J. D. Jackson, Am. J. Phys. 64, 855 (1996).

[19] J. D. Jackson, Classical Electrodynamics (Wiley, New York, 1975), 2nd ed. 